\title{
A CAMP-specific phosphodiesterase (PDE8B) that is mutated in adrenal hyperplasia is expressed widely in human and mouse tissues: a novel PDE8B isoform in human adrenal cortex
}

\author{
Anelia Horvath*,1 ${ }^{*}$ Christoforos Giatzakis ${ }^{1}$, Kitman Tsang ${ }^{1}$, Elizabeth Greene ${ }^{1}$, \\ Paulo Osorio ${ }^{1}$, Sosipatros Boikos ${ }^{1}$, Rossella Libè ${ }^{2}$, Yianna Patronas ${ }^{1}$, Audrey Robinson-White ${ }^{1}$, \\ Elaine Remmers ${ }^{3}$, Jerôme Bertherat ${ }^{2}$, Maria Nesterova ${ }^{1}$ and Constantine A Stratakis ${ }^{1}$
}

\begin{abstract}
${ }^{1}$ Section on Endocrinology \& Genetics, Program on Developmental Endocrinology \& Genetics, National Institute of Child Health and Human Development, National Institutes of Health, Bethesda, MD, USA; ${ }^{2}$ Departement Endocrinologie, Metabolisme and Cancer, Institut National de la Sante et de la Recherche Medicale U567, Institut Cochin, and Centre National de la Recherche Scientifique UMR 8104, and Centre de Reference des Maladies Rares de la Surrenale, Service d'Endocrinologie, Hôpital Cochin, Universite Paris 5, Paris, France; ${ }^{3}$ Genetics and Genomics Branch, National Institute of Arthritis and Musculoskeletal and Skin Diseases, NIH, Bethesda, MD, USA
\end{abstract}

Bilateral adrenocortical hyperplasia (BAH) is the second most common cause of corticotropin-independent Cushing syndrome (CS). Genetic forms of BAH have been associated with complex syndromes such as Carney Complex and McCune-Albright syndrome or may present as isolated micronodular adrenocortical disease (iMAD) usually in children and young adults with CS. A genome-wide association study identified inactivating phosphodiesterase (PDE) 11A (PDE11A)-sequencing defects as low-penetrance predisposing factors for iMAD and related abnormalities; we also described a mutation (c.914A $>C / H 305 P)$ in cyclic AMP (CAMP)-specific PDE8B, in a patient with iMAD. In this study we further characterize this mutation; we also found a novel $P D E 8 B$ isoform that is highly expressed in the adrenal gland. This mutation is shown to significantly affect the ability of the protein to degrade CAMP in vitro. Tumor tissues from patients with iMAD and no mutations in the coding PDE8B sequence or any other related genes (PRKAR1A, PDE11A) showed downregulated PDE8B expression (compared to normal adrenal cortex). Pde8b is detectable in the adrenal gland of newborn mice and is widely expressed in other mouse tissues. We conclude that PDE8B is another PDE gene linked to iMAD; it is a candidate causative gene for other adrenocortical lesions linked to the CAMP signaling pathway and possibly for tumors in other tissues.

European Journal of Human Genetics (2008) 16, 1245-1253; doi:10.1038/ejhg.2008.85; published online 23 April 2008

Keywords: adrenal gland; Cushing syndrome; protein kinase A; cyclic AMP; phosphodiesterases

*Correspondence: Dr A Horvath, SEGEN, NIH/NICHD, Building 10 Center Dr, CRC, Room 1E-3216, Bethesda, MD 20892, USA.

Tel: + 1301496 4686/496 6683; Fax: + 1301301402 0574/480-0378; E-mail: horvatha@mail.nih.gov

Received 5 January 2008; revised 11 March 2008; accepted 14 March 2008; published online 23 April 2008
Introduction

Micronodular adrenocortical disease (MAD) is a distinct type of corticotropin-independent bilateral adrenocortical hyperplasia (BAH) and is associated with Cushing syndrome (CS) in children and young adults. ${ }^{1}$ MAD is characterized by multiple nodules, capsular deficits that often result in extra-adrenal cortical excrescences 
and hyperplasia of the main cortical tissue; the adrenal medulla is normal in this condition. ${ }^{1-3}$ A rare variant of MAD is heavily pigmented due to the accumulation of lipofuscin in the cortical cells; this form is known as primary pigmented nodular adrenocortical disease (PPNAD). ${ }^{1,3}$ All forms of $\mathrm{BAH}$ have been linked to abnormalities of the cyclic AMP (cAMP) signaling pathway: ${ }^{4-6}$ GNAS is mutated in $\mathrm{BAH}$ in the context of McCune-Albright syndrome, ${ }^{4}$ and PRKAR1A mutations cause sporadic and familial PPNAD, the latter as part of Carney complex. ${ }^{5}$

Recently, a single-nucleotide polymorphism-based genome-wide association (GWA) study identified inactivating $P D E 11 A$-sequencing defects in 7 out of 17 patients with iMAD. ${ }^{7}$ PDE11A encodes a dual-specificity PDE that binds both cAMP and cGMP, and is expressed in a number of endocrine tissues, including the adrenal cortex..$^{7-11}$ Adrenocortical hyperplasia in the presence of a partially inactivated PDE is likely to be caused by high cellular cAMP levels in a manner analogous to endocrine tumor formation in McCune-Albright syndrome. ${ }^{7,12}$

The GWA study identified a number of other chromosomal loci as potentially linked to the development of iMAD. Among them, the chromosomal locus 5q13 containing the gene for a cAMP-specific PDE, PDE8B, was the second most favored by all analyses, including loss of heterozygosity and transmission disequilibrium testing (Horvath et al $(2006)^{7}$, supplementary data available at http://www. nature.com/ng/archive/suppinfo/index.html). PDE8B was also shown to have significantly higher expression in the adrenal gland compared to all other cAMP-specific PDEs, including PDE1A, PDE4A, PDE4B, PDE4C, PDE4D, PDE7A and PDE9A (Horvath et al (2006) ${ }^{7}$, supplementary data). We then identified a single germline $P D E 8 B$ missense substitution (c.914A $>$ C/H305P) in a patient with iMAD, which we reported as a brief case report. ${ }^{13}$

In the present report, we expand this observation on the identified $P D E 8 B$ mutation and the involvement of this gene in adrenal hyperplasias; we characterized further the mutation and identified a novel PDE8B isoform that is expressed in adrenocortical and other tissues, and, finally, examined $P D E 8 B$ expression in the developing and adult mouse tissues.

\section{Materials and methods}

\section{Subjects and their samples}

The institutional review boards of NICHD, NIH, approved the genetic investigation of patients with adrenocortical tumors under NICHD protocols 95-CH-0059 and 00-CH0160 after the patients had given informed consent; 22 unrelated pediatric patients with isolated $\mathrm{BAH}$ were included in this study, including the 17 patients described previously. ${ }^{7}$ The affected individuals ranged in age from 1 to 14 , with an average age of 6.2 years. Patients who were diagnosed with CS by standard clinical criteria and testing underwent adrenalectomy. The controls included in this study have been described elsewhere. ${ }^{7,14}$

DNA, RNA, protein extraction and sequence analysis Blood and tissue samples were collected, as previously described. ${ }^{14}$ DNA, RNA and proteins were extracted from whole blood and cell cultures using standard procedures.

All the primers used for the PCR amplification of the PDE8B coding regions and exon-intron junctions are listed in Table 1. After the amplification, the PCR products were agarose gel purified (Minelute; Qiagen) and bidirectionally sequenced on $3130 \times 1$ Genetic Analyzer (Applied Biosystems). Sequences were analyzed using Vector NTI 10 software (Invitrogen).

\section{5'-RACE}

Human adrenal total RNA (Ambion) was subjected to RNA ligase-mediated 5'-RACE (RLMRACE) using the GeneRacer (Invitrogen) kit according to the manufacturer's instructions. Briefly, $1 \mu \mathrm{g}$ total RNA was reverse transcribed with Superscript III RT (Invitrogen), and cDNA was subjected to two successive rounds of PCR with Accuprime Taq polymerase (Invitrogen) using primer R3: CACTGGGGTGAT CTTCACG-TGCTGTTGG and nested primer R2: CCTGCCA CTCCTTTCCCTTCTTGATGCAT. The resulting amplimer was TA-subcloned and fully sequenced.

\section{Generation of PDE8B c.914C > A substitution-bearing constructs}

Wild-type PDE8B1 (Origene) was used to first generate the isoform that was identified in the adrenal gland. The endonucleases EcoRI and ApaI were used according to the manufacturer's instructions (New England Biolabs). The first exon was excited from PDE8B1 and replaced with the newly identified exonic sequence from our RACE products. Further, c.914C $>$ A substitution was introduced using overlapping PCR with the following primers:

F1: CAGTGTTTACAGCATTAGATCACTGTC;

R1: CAGACAGAGCCTCCTTCATTCAGATAT;

F2: ATATCTGAATGAAGGAGGCTC-TGTCTG;

R2: ATCTCTAGAACTCTGTCCAAGGCTTC.

Transfection experiments were performed as previously described. ${ }^{14}$ cAMP levels were measured by cAMP-Glo Assay (Promega) as recommended by the manufacturer.

\section{Mouse expression studies}

Pde8b mice expression studies were performed in collaboration with Phylogeny (www.phylogenyinc.com). Tissues were obtained from e9.5, e15.5, p1, p10 and adult mice. Frozen tissues were cut into 8 - to $10-\mu \mathrm{m}$ sections, fixed for $60 \mathrm{~min}$ in cold acetone, air-dried and then stored at room temperature. The antibody used in this study (rabbit polyclonal antibody to human PDE8B 
Table 1 Primers used for PDE8B sequencing

\begin{tabular}{|c|c|c|}
\hline Exon no. & Forward primer sequence $\left(5^{\prime}-3^{\prime}\right)$ & Reverse primer sequence $\left(5^{\prime}-3^{\prime}\right)$ \\
\hline 1 & gaggaagatggcccaaaag & gctcccatcatctccacaaa \\
\hline 2 & agtgcacacggtggcataat & gagccgagactgagcctcta \\
\hline 3 & gctgggattacaggcatgag & ggagagagaaatctacaggaagga \\
\hline 4 & tcctaatccacaagggcatc & aасаaаcaаaасcсcсаaаg \\
\hline 5 & ctgctggagctttctctgct & gtcctggggcttaatttcct \\
\hline 6 & caagcactttgaacaccttga & tgccattcattgcctgttta \\
\hline 7 & ttgggagacatcagcattca & tcagtattctttgcacagcttga \\
\hline 8 & cttcctacggggcacaca & ccagattcacttgagttccaaa \\
\hline 9 & aggcattgggaaatgtaacg & gctcatactggcatttcaagc \\
\hline 10 & atgtgtgggctctgtgtgaa & aaagatttgtcagaggaaccaaa \\
\hline 11 & tqgtgtatgtctttcatctgttca & tgccataaagqaagattcaagg \\
\hline 12 & gcccggcctaatgtttattt & ccctccaaagagacgacaaa \\
\hline 13 & ctcctgcctccaaagttcc & ctctcacagaacccgcttg \\
\hline 14 & tcttttggattctgggcata & cagcctcatggaaagacaaa \\
\hline 15 & atcccagttctccacgttct & tgcaattcttactctaactgtgctc \\
\hline 16 & cttccagctagtcccatttga & cagqgaccatagtctctctg \\
\hline 17 & cccctgtgtgtgtgaagcta & tggcagaaaggtccatgtc \\
\hline 18 & cqcctctccacatctaggtc & gaactcactgaagaccaaatgagat \\
\hline 19 & ctggggaaaatggaatagca & ttcatccccaaggaaaacaa \\
\hline 20 & acaggtggtgacagggactt & aaggaaccagaagcctaccc \\
\hline 21 & aggcttgagagtgqgaaggt & ctgctgaggatgcaaagaat \\
\hline $22 \mathrm{~A}$ & gatcccaaacttgtcccaga & CAGGATGACAGCAGAGCAAA \\
\hline $22 \mathrm{~B}$ & GTTTGAGGCTTCCATCTGACA & AGCCATAGAGGCTGTGAAGC \\
\hline $22 \mathrm{C}$ & САТСТСССАGGATGGTGACT & ACTTGGTGTAGTGCCTCGTG \\
\hline 22D & СТTCTGGCCGATGGTATGAC & ccctatcccctcccaaaat \\
\hline
\end{tabular}

${ }^{a}$ Capital letters indicate exonic primers.

synthetic peptide) was purchased from Abcam (catalog no. ab14621). This antibody has no cross-reactivity with PDE8A or any other PDE family members. Slides were washed in PBS and then incubated with antibody and revealed according to peroxidase technique. To detect nonspecific immunoreactivity, normal rabbit serum was used as a control.

To perform IHC, the sections were hydrated in PBS (from Invitrogen $10 \times, \mathrm{pH} 7.2$ ), blocked in TBST (TrisBuffered Saline with Tween 20, Dako catalog no. S3006) for $5 \mathrm{~min}$, peroxidase-blocked $\left(0.03 \% \mathrm{H}_{2} \mathrm{O}_{2}\right.$ in $\left.\mathrm{diH}_{2} \mathrm{O}\right)$ for $5 \mathrm{~min}$ and rinsed (20 dips) in PBS. PDE8B antibody diluted 1:50 with antibody diluent (Dako catalog no. S0809) was applied to the sections for $60 \mathrm{~min}$ at room temperature, then rinsed with PBS for $5 \mathrm{~min}$. Avidin/biotin blocking solution (Dako catalog no. X0590) was applied for $20 \mathrm{~min}$ followed by a PBS rinse for $5 \mathrm{~min}$. Goat anti-rabbit antibody (Vector BA-1000) was applied at a dilution of 1:200 for $20 \mathrm{~min}$ at room temperature followed by a rinse with PBS for $5 \mathrm{~min}$. The detection reagent Vectastain Elite (Vector PK-6100) was applied for $30 \mathrm{~min}$ and DAB (Dako catalog no. K3468) for $5 \mathrm{~min}$ at room temperature. This procedure produced brown immunostaining within cell cytoplasm or around cells. The sections were rinsed in $\mathrm{diH}_{2} \mathrm{O}$, counterstained with hematoxylin, rinsed again in $\mathrm{diH}_{2} \mathrm{O}$, dehydrated in a series of alcohols and xylene and then coverslipped. All sections were counterstained with hematoxylin and photographed under brightfield illumination.

\section{Results \\ PDE8B mutation analysis in patients with iMAD and CS} A total of 22 subjects with iMAD and CS were studied, of whom 18 were Caucasians, two African-Americans, one Hispanic and one South Asian (from Pakistan). All samples were negative for PRKAR1A, MYH8 and GNAS mutations (data not shown). This cohort included the 17 patients screened for the presence of PDE11A-inactivating mutations and has been described previously. ${ }^{7}$ The single base substitution (c.914A $>\mathrm{T} / \mathrm{H} 305 \mathrm{P}$ ) that was reported elsewhere $^{13}$ was found in the linker region between the PAS (period, aryl-hydrocarbon receptor nuclear translocator (ARNT) and single-minded) and the catalytic domain of PDE8B in a female patient with CS (CAR 559.03). As we reported elsewhere, the patient had presented in early childhood with obesity, hypercholesterolemia and growth abnormalities due to iMAD; $^{13}$ she had inherited the mutation from her father (CAR 559.01), who was not known to have CS, but whose subsequent investigation revealed a phenotype consistent with mild iMAD: he had abnormal midnight cortisol levels, ${ }^{13}$ obesity, hypertension and, on computed tomography, mild $\mathrm{BAH}$, more prominent on the left adrenal gland (Figure 1). The c.914A $>\mathrm{T}$ $P D E 8 B$ mutation has not been found in 1030 unrelated control individuals from a variety of populations, including 285 controls from a mostly Latin-American-based population sample (Coriell Institute; http://www.coriell.org/) and 745 individuals enrolled in the New York Cancer Project (NYCP) study. ${ }^{14}$ 


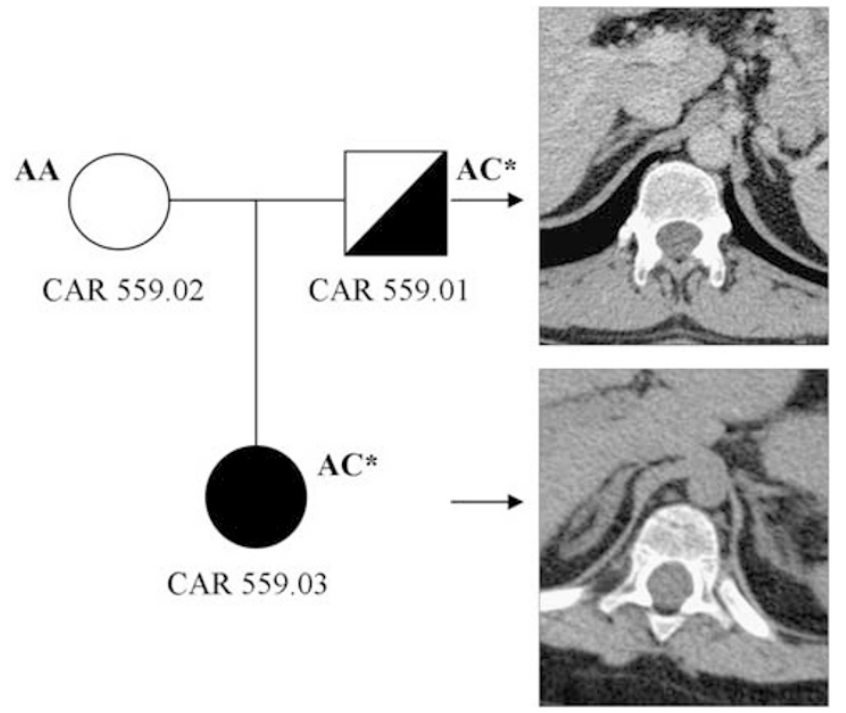

Figure 1 Computed tomography (CT) of the adrenal gland of our patient (CAR 559.03) and her father (CAR 559.01), who is also carrier of the H305P mutation in PDE8B.

\section{In vitro studies of the effect of H305P mutation; novel PDE8B isoform}

The H305P mutation affects an evolutionarily conserved residue of the PDE8B protein (Figure 2a). To estimate the effect on the protein function, we performed in vitro studies on HEK293 cells: the c.914A $>\mathrm{T}$ substitution led to significantly higher cAMP level in cells transfected with the PDE8B bearing the substitution. ${ }^{13}$

During our attempts to amplify the whole open reading frame (ORF) of PDE8B from adrenal tissue, we identified the presence of an isoform shorter than the previously published one with a different $5^{\prime}$-end. We performed $5^{\prime}$ RACE and isolated a distinct, novel isoform (Figure 2b). The novel isoform includes one additional $5^{\prime}$-located exon of $53 \mathrm{bp}$ that contains an in-frame initiation codon, accompanied by classical Kozak motif; the deduced protein sequence of 12 aa is presented in Figure 2c. This exon was located $30473 \mathrm{bp} 5^{\prime}$ of the known transcription start site, and its upstream sequences displayed clear promoter and regulatory features. Similar to the already known transcription initiation site, a CpG island was located between positions -245 and 454 of the newly identified initiator codon. In contrast to all the known PDEBB isoforms, the novel isoform skips the currently recognized exon 1 , known as the longest exon of the gene (Figure 2d). Sequencing of the newly identified exon in our 22 patients did not reveal any alterations from the published genomic sequence (data not shown). Quantitative PCR showed highest expression of the novel isoform in the pancreas, followed by prostate, testis, heart, kidney, ovary and adrenal (Figure 2e). The ability of the novel isoform to degrade cAMP was similar to PDE8B1, as shown by our transfection experiments on HEK293 cells; this was compared with the wild-type, known ORF and the mutant c. $914 \mathrm{~A}>\mathrm{T}$ form (Figure 3).

\section{PDE8B expression in normal and a series of adrenocortical tumors}

We assessed PDE8B expression in a panel of three normal and six adrenocortical tumor samples using western blot analysis. These samples have been screened for CTNNB1-, GNAS-, PDE11A- and PDE8B-sequence defects and none was found (data not shown). All six samples contained lower PDE8B protein levels despite the absence of mutations in the coding sequence of $P D E 8 B$ (Figure 4). Interestingly, quantitative PCR on the same adrenocortical samples did not show differential PDE8B expression between normal and tumor tissues (data not shown).

\section{Pde8b protein expression in mice}

We studied $P d e 8 b$ protein expression in embryonic and adult mice; a summary of Pde8b distribution at stages e15.5, p1, p10 and p77 is presented in Table 2. PDE8b was undetectable at stage e9.5 in the embryo, but occurs in e15.5 mouse tissues (Figure 5a). At this stage, Pde8b was found primarily in the embryonic adipose tissue primordium forming the future hibernation gland that is located in the space between the scapulae (Figure 5b). Little Pde8b was found in the e15.5 liver and heart ventricle (Figure 5c and $\mathrm{d}$ respectively). Pde8b distribution formed islets around groups of liver cells and between the heart ventricle muscle fibers; this type of extracellular labeling was not seen in other regions.

Relative to the embryonic stage, a much greater amount of Pde8b was detected in the p1 mouse. Pde8b was labeled in the adipose tissue, especially in the hibernating gland situated between the scapulae (Figure 5e). Also evident was the presence of Pde $8 \mathrm{~b}$ in the heart and liver, stomach mucosa, and in the convoluted tubules of the kidney (Figure $5 \mathrm{f}-\mathrm{i}$ ). The way the labeling is distributed in these organs (around, rather than within the cells) suggests the presence of Pde8b on cell surfaces. Pde8b was detected on the surface of cardiomyocytes, the epithelial-cell apical portion of the stomach and the brush border surface of the presumptive kidney distal tubules. Alternatively, this pattern of labeling may be attributable to the presence of Pde8b in the interstitial space between cells.

By postnatal day 10, a decrease of Pde8b was observed in many tissues, including the adipose tissue (Figure $5 \mathrm{j}$ ). Taking into account mass and distribution, however, the adipose tissue still can be considered to contain more Pde8b than any other tissue. Lower Pde8b levels were detected in the heart ventricle tissue.

In the liver, the pattern of Pde8b changed from extracellular in the embryo to intracellular in the adult (Figure 5k). Barely detectable Pde8b was seen in the epithelium of the apical portion of the lung bronchioles 
a

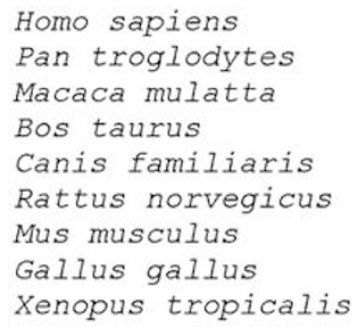

IHKIHRDSGDNSQTEP ISSRYKNRRKESIDVKS IHKIHRDSGDNSQTEPHSFRYKNRRKES IDVKS IHKIHRDSGDNSQTEPHSFRYKNRRKESIDVKS I LKIHRDSGDNSQTESHSFRYKNRRKESIDVKS +HK+HRDS DNSQTE HSFRYKNRRKESIDVKS $I H+I H R D S G D N S Q T E P H S F R+K+R R K E S I D V K S$ IH+IHRDSGDNSQTEPHSFR+KNRRKES IDVKS HRD SGDNSQ+E HSF+ KNRRK+SIDVKS QKHLHLYFECLFLSESHSFRLKNRRKESVDVRS

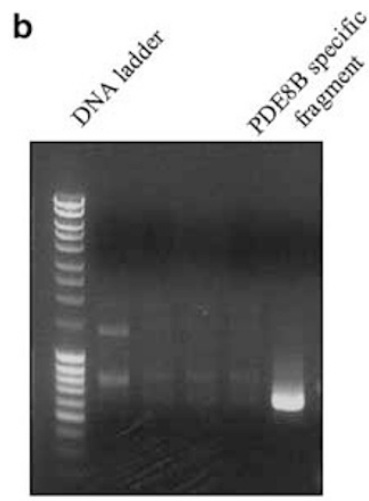

C

\begin{tabular}{|lccccccccccccc|}
\hline Sequence & AGCCGCCCGCCGCCG & ATG & GAC & CAG & CTG & TAC & TGC & CCA & CCG & AGC & GCG & TGC & CAG \\
\hline AA & M & D & Q & L & Y & C & P & P & R & A & C & Q \\
\hline
\end{tabular}

d

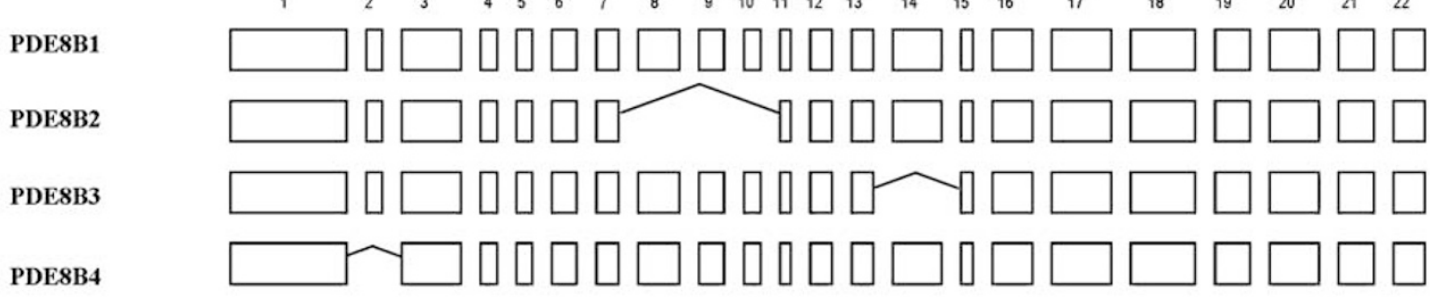

PDE8B6

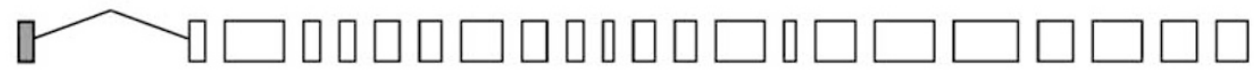

e
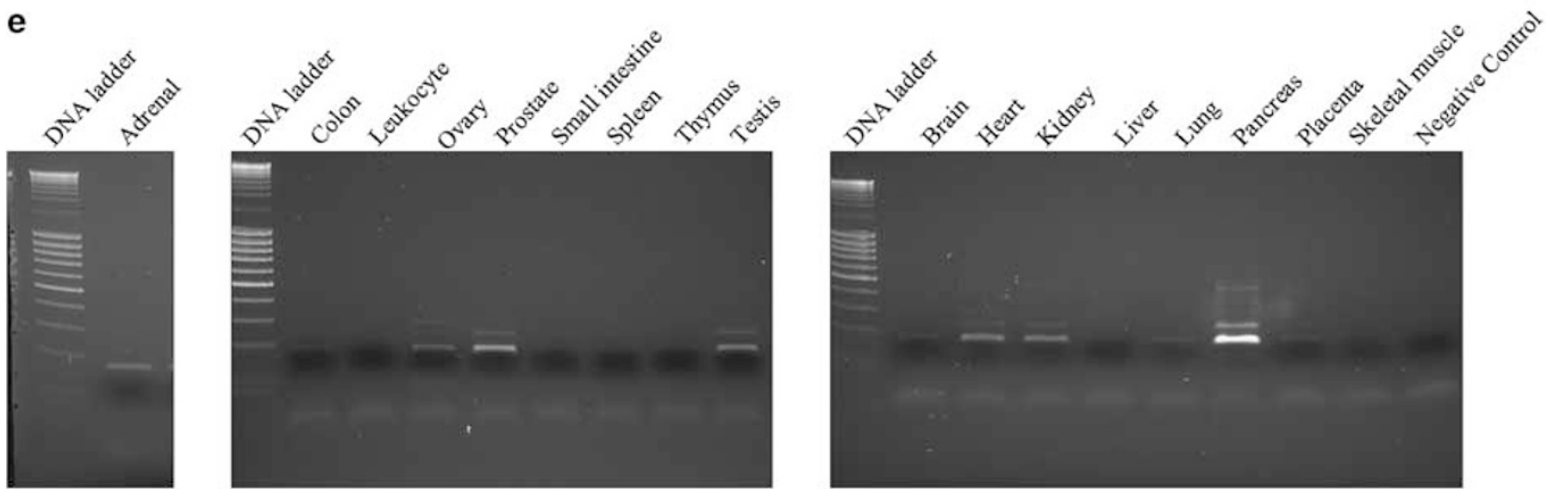

Figure 2 (a) Conservation of His at position 305 of PDE across different species; (b) gel electrophoresis on the products generated by 5'-RACE; the adrenal-specific fragment that was further used to identify the $5^{\prime}$-end of the novel isoform is shown; (c) nucleotide and amino-acid sequence of the novel exon; (d) PDE8B isoforms generated by alternative splicing; the newly identified exon is shown in red; (e) expression of the novel isoform across panel of 17 normal human tissues.

(Figure 5l). The pancreas exhibited faint labeling in the endocrine pancreatic islets and even less in the exocrine pancreatic acini (Figure $5 \mathrm{~m}$ ). There was detectable Pde8b in the vas deferens (data not shown). In the male reproductive organs, detectable Pde8b occurred in the seminiferous tubules of the testis, consistent with the distribution of the spermatogonia, but not spermatozoa (Figure $5 \mathrm{n}$ ).

\section{Discussion}

The reported $\mathrm{P} 305 \mathrm{H}$ substitution in PDE8B was present in family members with very mild to a clinically significant phenotype in terms of the severity of $\mathrm{CS}^{13}$ We have reported before similar differences in the phenotype of iMAD; $;, 14$ it appears that PDE-sequence defects are acting as predisposing factors to the development of nodularity 


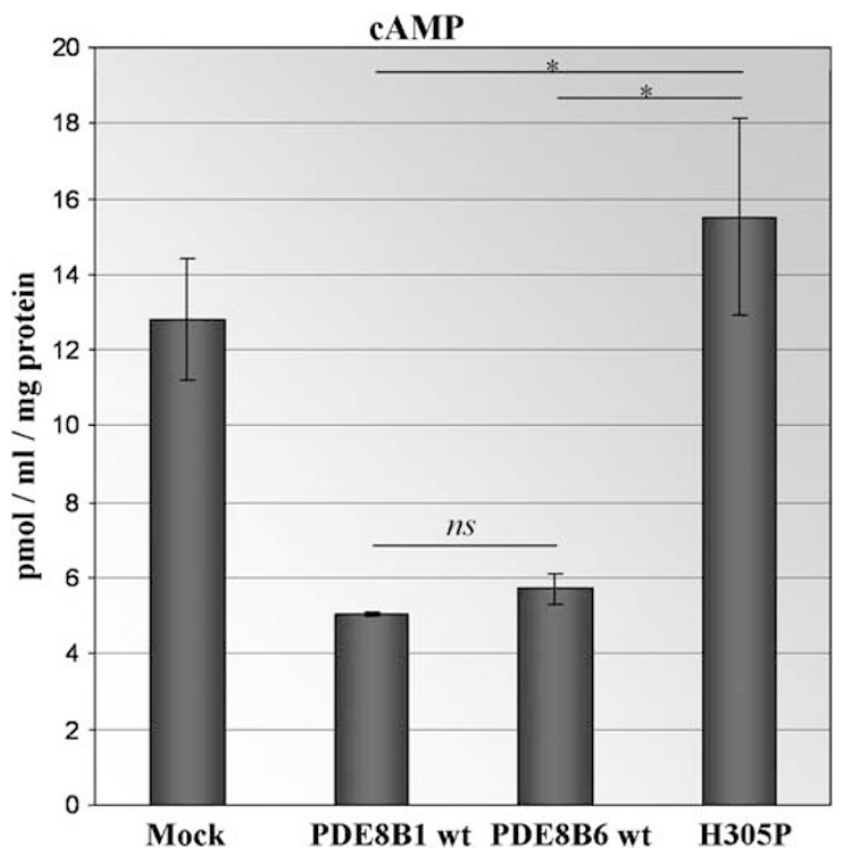

Figure 3 In vitro experiments with PDE8B ORF construct; asterisk indicates significance of the observed difference between the wild-type and mutant constructs $(P<0.05)$. and they are not causative per se. This is consistent with the relatively high (for a rare disease) frequency of $P D E 11 A$ sequencing defects in the general population, ${ }^{14}$ although the $\mathrm{P} 305 \mathrm{H} P D E 8 B$ mutation was not found in more than 2000 control chromosomes.

Although the cause of all forms of BAH studied to date seems to be linked to increased cAMP signaling, the histopathological changes in the adrenal glands of patients with the various mutations or functional abnormalities of this pathway differ significantly and overlap only partially. ${ }^{1}$ PRKAR1A mutations are associated with PPNAD, ${ }^{5}$

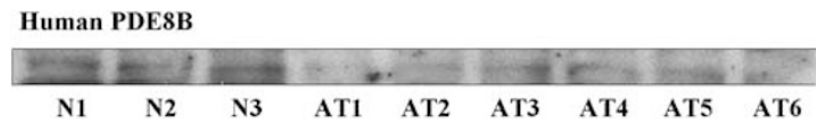

Human beta-actin

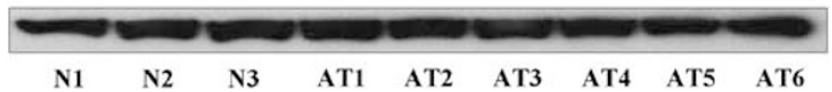

Figure 4 Western blot analysis of PDE8B in three normal $(N)$ and six tumor adrenocortical (AT) tissue samples from patients with no mutations in the coding sequence of PDE8B. Lower protein levels in the tumor samples compared to the normal tissues were observed. The protein expression is controlled by $\beta$-actin.

Table 2 IHC distribution of PDE8B

\begin{tabular}{|c|c|c|c|c|}
\hline Tissue & e15.5 (sex not determined) & p1 & p10 & $p 77$ \\
\hline Adipose tissue axillae (M) & $+++++/-$ & $+++++/-$ & $+++/-$ & ne \\
\hline Adipose tissue axillae (F) & & $+++++/-$ & $+++/-$ & $++/-$ \\
\hline Adipose tissue scapulae (M) & $++/-$ & $+++++/-$ & $+++/-$ & $++/-$ \\
\hline Adipose tissue scapulae (F) & & $+++++/-$ & $++++/-$ & $+++/-$ \\
\hline Adrenal gland $(\mathrm{M})$ & ne & $+1-$ & ne & ne \\
\hline Adrenal gland (F) & & $+1-$ & ne & ne \\
\hline Adipose around blood vessels (M) & $-1-$ & $++/-$ & $+++/-$ & $++/-$ \\
\hline Adipose around blood vessels (F) & & $+++/-$ & $+++/-$ & $++/-$ \\
\hline Brain (M) & $+1-$ & $+1-$ & $+/+$ & ne \\
\hline Brain $(F)$ & & $+1-$ & $+/+$ & ne \\
\hline Stomach (M) & - & $++/-$ & $+/+$ & ne \\
\hline Stomach (F) & & $+++/-$ & $+/+$ & ne \\
\hline Small intestine (M) & $-1-$ & $+++/-$ & $++1-$ & ne \\
\hline Small intestine $(\mathrm{F})$ & & $+++/-$ & $+++/-$ & +++ \\
\hline Heart, ventricle (M) & $+1-$ & $++1-$ & $+1-$ & $-1-$ \\
\hline Heart, ventricle (F) & & $++/-$ & $+1-$ & $+1-$ \\
\hline Kidney (M) & $++/-$ & $++/-$ & ne & ne \\
\hline Kidney (F) & & $+++/-$ & ne & ne \\
\hline Liver (M) & $+++/-$ & $++/-^{\mathrm{a}}$ & $++/-$ & $+++/-$ \\
\hline Liver $(F)$ & & $++/-^{a}$ & $++/-$ & $+++/-$ \\
\hline Lung, bronchus (M) & $+1-$ & $+1-$ & $-1-$ & $-1-$ \\
\hline Lung, bronchus (F) & & $+1-$ & $+1-$ & $-1-$ \\
\hline Skeletal muscles (M) & $+1-$ & $-1-$ & $+1-$ & $-1-$ \\
\hline Skeletal muscles (F) & & $+1-$ & $+1-$ & $-1-$ \\
\hline Pancreatic islets (M) & ne & $-1-$ & $-1-$ & ne \\
\hline Pancreatic islets (F) & & $-1-$ & $-1-$ & $++/-$ \\
\hline Spleen (M) & ne & $+/-$ & ne & ne \\
\hline Spleen (F) & & $++/-$ & ne & $+1-$ \\
\hline Testis & $+1-$ & \pm & $+/-$ & $-1-$ \\
\hline
\end{tabular}

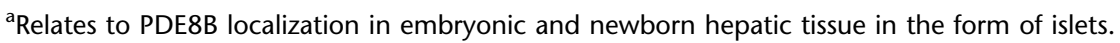

Score: ne, not examined; -, undetectable; +, weak; +++, medium; +++++, strong immunoreaction presented as PDE8B/lgG. 

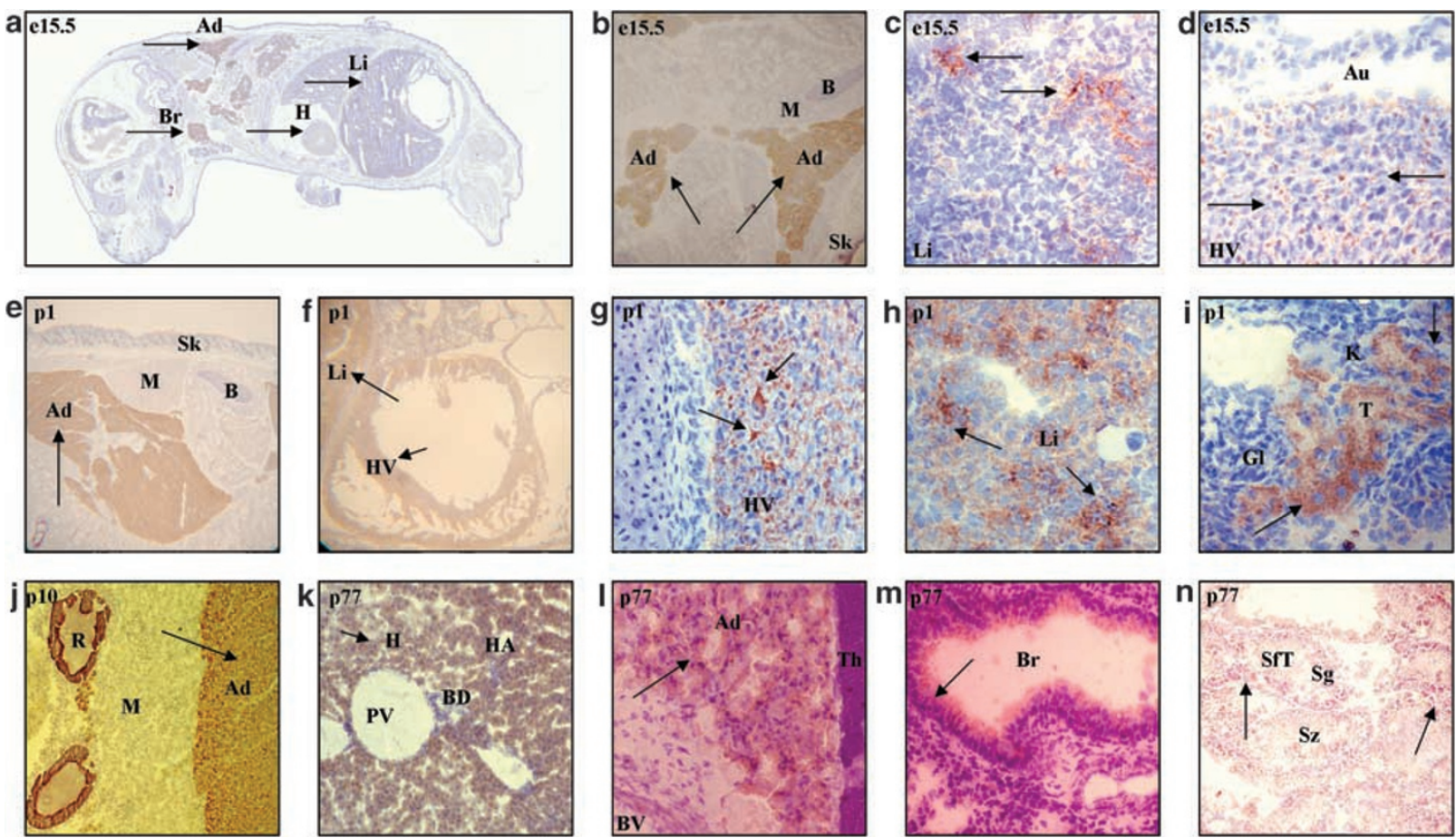

Figure 5 (a) Brown PDE8B immunostaining (arrows) in the mouse embryo on day 15.5, seen in a whole-body section ( $\mathrm{Br}$ - brain; $\mathrm{H}$ - heart; $\mathrm{Li}$ liver; Ad - adipose tissue, upper arrow indicates the hibernating gland); (b) brown PDE8B immunostaining (arrows) in the adipose tissue; upper arrow indicates the hibernating gland (B - bone; $\mathrm{M}$ - skeletal muscle; Sk - skin); (c) immunostaining in embryonic liver showing brown labeling forming the islets in between the liver cells (arrows); (d) PDE8B in the heart ventricle (HV) forming small spots between muscle fibers (arrows). The atria (Au) do not show any labeling; (e) brown PDE8B immunostaining in the adipose tissue of the hibernating gland of a newborn mouse, arrow indicates the hibernating gland; (f) PDE8B immunostaining (arrows) in the heart ventricle and liver of a newborn mouse; $(g)$ the wall of the heart ventricle; PDE8B labeling (arrow) forms a network around the muscle fibers; (h) liver; PDE8B labeling (arrow) seen as a heterogeneous distribution throughout the liver parenchyma; (i) kidney tubules, most likely distal tubules, but not glomeruli, showing PDE8B labeling (arrow); GI - glomerulus; $\mathrm{K}$ - kidney; T - tubules; (j) intense PDE8B immunostaining (arrow) in the adipose tissue; R - rib; (k) PDE8B immunostaining (arrow) in the liver parenchyma; blood vessels, including branches of the portal vein and hepatic artery, and bile duct remain unlabeled (BD - bile duct; $\mathrm{H}$ - hepatocyte; HA - hepatic artery; PV portal vein branch); (I) PDE8B immunostaining (arrow) in the brown adipose tissue surrounding the thymus; thymus and blood vessels remain unstained (BV - blood vessel; Th - thymus); (m) light PDE8B immunostaining (arrow) in the epithelial cells of the bronchioles (Br - bronchiole); ( $\mathrm{n}$ ) PDE8B immunostaining (arrow) in the seminiferous tubules of the adult mouse testis showing spermatogonia to be slightly labeled, spermatozoa to be unstained (SfT - seminiferous tubule; Sg - spermatogonia; Sz - spermatozoa).

whereas PDE11A and possibly PDE8B mutations seem to predispose to a variety of lesions from isolated (without any other associated tumors) PPNAD to nonpigmented iMAD and other forms of $\mathrm{BAH} ;{ }^{7,13,14}$ GNAS mutations are associated with the mostly macronodular and clearly nonpigmented form of BAH that one sees in McCuneAlbright syndrome. ${ }^{4}$ The identification of more mutations in these and other PDEs will shed more light on this apparent lack of tight genotype-phenotype correlation, which is somewhat unexpected as tissues affected by PPNAD are almost identical histologically.

PDE8B encodes cAMP-specific PDE with the highest affinity to cAMP among all the known PDEs. ${ }^{15,16}$ Although involvement in some environment signal transduction has been suggested by the presence of REC (receiver) and PAS domains, no ligand has yet been identified, and the function of PDE8B remains largely unknown. As in other PDEs, the PDE8B locus is quite complex and encodes multiple isoforms, arising mainly from alternative splicing and displaying tissue-specific expression., ${ }^{9-18}$ We describe here a novel isoform that is highly expressed in endocrine tissues, including the adrenal gland, where it shows the highest expression level compared to other cAMP-degrading PDEs. The high adrenal-specific expression levels, its significant affinity to cAMP and the overall importance of cAMP signaling for adrenocortical tumorigenesis suggest that PDE8B could be a candidate for further investigations of its role in adrenal (and possibly other endocrine) tumors.

We detected decreased PDE8B protein levels in adrenocortical tumor samples that are negative for germline mutations in the gene. The real-time PCR experiments did not show significant alteration of the PDE8B mRNA levels in the same tumors, thus suggesting posttranscriptional events involved in the regulation of expression. The human microRNA databases list four miRNAs that are 
predicted to target PDE8B: hsa-miR-412, hsa-miRNA-7705p, hsa-miRNA-520d-5p and hsa-miRNA-493 (www. microRNA.org). All four of them recognize 5 -located regions of the gene that do not overlap with the c.914A $>C$ substitution. Ongoing experiments assess the ability of those miRNAs to silence PDE8B expression in vitro.

Mouse studies showed that predominant expression of Pde8b in the adipose tissue was detected in both embryonic and adult mouse. At embryonic stage e15.5, Pde8b was labeled primarily in the adipose tissue primordium forming the future hibernation gland. Late in gestation, the hibernation gland accumulates fat to serve as an immediate energy reserve at birth. Although the hibernation gland does not yet contain fat at stage e15.5, the presence of Pde8b may indicate that it plays a role in processes related to fat intake and storage. The adipose remained to be the tissue with the highest Pde8b expression in newborn (p1) and adult mice (p10 and p77), although slight decrease in the protein levels has been observed after birth.

Besides its role in the energy intake and storage, the adipose tissue has been recently recognized to have an important endocrine function, and leptin has been the first characterized hormone that is secreted by the adipocytes. ${ }^{19}$ Several studies from the last decade have contributed to the elucidation of the endocrinology of adipose tissue and its secretion of adipokines. ${ }^{20,21}$ The early and stable Pde8b expression in mouse adipocytes is in need of further investigation. A recent study explores the stimulatory effect of adipose tissue-derived factors on adrenal steroidogenesis and secretory function. ${ }^{22}$ An important cross talk between adipose tissue and adrenocortical function is supported by the study of Lamounier-Zepter et $\mathrm{al}^{23}$ on AZIP/F1 transgenic mice. This and previous studies have suggested that leptin deficiency may be one of the causes for increased adrenal glucocorticoid production. ${ }^{24}$ As leptin signaling has been suggested to involve another cAMP-specific PDE, PDE3B, a similar role could be speculated for Pde8b. ${ }^{25}$

Apart from the adipose tissue, an early Pde8b labeling was detected in the liver and the heart ventricle. The type of labeling of Pde8b in these two tissues was suggestive of extracellular or membranous localization of the protein. The same localization of Pde $8 \mathrm{~b}$ was observed later at $\mathrm{p} 1$ stage in the newborn mouse liver and heart where, however, significantly higher levels of the protein were detected. In addition, at p1 stage, Pde $8 \mathrm{~b}$ was detected on the surface of the epithelial-cell apical portion of the stomach and the brush border surface of the presumptive kidney distal tubules. At p10 stage, a slight decrease in the Pde8B expression was detected in almost all of the tissues. Considering ontogeny, differences in the subcellular Pde8b localization were noted, especially in the liver. In the embryonic and newborn mouse liver, Pde8b seemed to exhibit a rather membranous (or extracellular) localization pattern, whereas in the adult mouse liver, Pde8B was localized in the cytoplasm. This observation requires further specifically designed subcellular localization studies including in situ hybridization to define the cells that are expressing the gene during the time when extracellular localization is observed.

In conclusion, we describe here the expression studies of a mutation of $P D E 8 B$ causing iMAD and the identification of a novel expression isoform of this gene that is expressed in the adrenal gland. We also present expression studies for this gene in normal mouse tissues; these studies suggest a wider role of PDE8B in endocrine function.

\section{Acknowledgements}

It was supported by US NIH intramural project Z01-HD-000642-04 to CAS and, in part, by Groupement d'Intérêt Scientifique-Institut National de la Santé et de la Recherche Médicale Institut des Maladies Rares and the Plan Hospitalier de Recherche Clinique (AOM 02068) to the Comete Network.

\section{Conflict of interest}

The authors declare that they have no competing financial interests.

\section{References}

1 Stratakis CA, Boikos SA: Genetics of adrenal tumors associated with Cushing's syndrome: a new classification for bilateral adrenocortical hyperplasias. Nat Clin Pract Endocrinol Metab 2007; 3: 748-757.

2 Gunther DF, Bourdeau I, Matyakhina L et al: Cyclical Cushing syndrome presenting in infancy: an early form of primary pigmented nodular adrenocortical disease, or a new entity? J Clin Endocrinol Metab 2004; 89: 3173-3182.

3 Stratakis CA: Adrenocortical tumors, primary pigmented adrenocortical disease (PPNAD)/Carney complex, and other bilateral hyperplasias: the NIH studies. Horm Metab Res 2007; 39: 467-473.

4 Weinstein LS, Yu S, Warner DR, Liu J: Endocrine manifestations o stimulatory $\mathrm{G}$ protein-subunit mutations and the role of genomic imprinting. Endocr Rev 2001; 22: 675-705.

5 Kirschner LS, Carney JA, Pack SD et al: Mutations of the gene encoding the protein kinase A type I-alpha regulatory subunit in patients with the Carney complex. Nat Genet 2000; 26: 89-92.

6 Bourdeau I, Stratakis CA: Cyclic AMP-dependent signaling aberrations in macronodular adrenal disease. Ann N Y Acad Sci 2002; 968: 240-255.

7 Horvath A, Boikos S, Giatzakis C et al: A genome-wide scan identifies mutations in the gene encoding phosphodiesterase 11A4 (PDE11A) in individuals with adrenocortical hyperplasia. Nat Genet 2006; 38: 794-800.

8 Fawcett L, Baxendale R, Stacey P et al: Molecular cloning and characterization of a distinct human phosphodiesterase gene family: PDE11A. Proc Natl Acad Sci USA 2000; 97: 3702-3707.

9 Yuasa K, Kotera J, Fujishige K, Michibata H, Sasaki T, Omori K: Isolation and characterization of two novel phosphodiesterase PDE11A variants showing unique structure and tissue-specific expression. J Biol Chem 2000; 275: 31469-31479.

10 Hetman JM, Robas N, Baxendale R et al: Cloning and characterization of two splice variants of human phosphodiesterase 11A. Proc Natl Acad Sci USA 2000; 97: 12891-12895.

11 Loughney K, Taylor J, Florio VA: 3',5'-cyclic nucleotide phosphodiesterase 11A: localization in human tissues. Int J Impot Res 2005; 17: $320-325$. 
12 Diaz A, Danon M, Crawford J: McCune-Albright syndrome and disorders due to activating mutations of GNAS1. J Pediatr Endocrinol Metab 2007; 20: 853-880.

13 Horvath A, Mericq V, Stratakis CA: Mutation in PDE8B, a cAMPspecific phosphodiesterase in adrenal hyperplasia. $N$ Engl $J$ Med 2008; 358: 750-752.

14 Horvath A, Giatzakis C, Robinson-White A et al: Adrenal hyperplasia and adenomas are associated with inhibition of phosphodiesterase $11 \mathrm{~A}$ in carriers of PDE11A sequence variants that are frequent in the population. Cancer Res 2006; 66: $11571-11575$.

15 Soderling SH, Bayuga SJ, Beavo JA: Cloning and characterization of a cAMP-specific cyclic nucleotide phosphodiesterase. Proc Natl Acad Sci USA 1998; 95: 8991-8996.

16 Hayashi M, Shimada Y, Nishimura Y, Hama T, Tanaka T: Genomic organization, chromosomal localization, and alternative splicing of the human phosphodiesterase 8B gene. Biochem Biophys Res Commun 2002; 297: 1253-1258.

17 Gamanuma M, Yuasa K, Sasaki T, Sakurai N, Kotera J, Omori K: Comparison of enzymatic characterization and gene organization of cyclic nucleotide phosphodiesterase 8 family in humans. Cell Signal 2003; 15: 565-574.

18 Conti M, Beavo J: Biochemistry and physiology of cyclic nucleotide phosphodiesterases: essential components in cyclic nucleotide signaling. Annu Rev Biochem 2007; 76: 481-511.
19 Zhang Y, Proenca R, Maffei M, Barone M, Leopold L, Friedman JM: Positional cloning of the mouse obese gene and its human homologue. Nature 1994; 372: 425-432.

20 Kim S, Moustaid-Moussa N: Secretory, endocrine and autocrine/ paracrine function of the adipocyte. J Nutr 2000; 130: 3110S-3115S

21 Trayhurn P, Beattie JH: Physiological role of adipose tissue: white adipose tissue as an endocrine and secretory organ. Proc Nutr Soc 2001; 60: 329-339.

22 Ehrhart-Bornstein M, Lamounier-Zepter V, Schraven A et al: Human adipocytes secrete mineralocorticoid-releasing factors. Proc Natl Acad Sci USA 2003; 100: 14211-14216.

23 Lamounier-Zepter V, Bornstein SR, Kunes J et al: Adrenocortical changes and arterial hypertension in lipoatrophic A-ZIP/F-1 mice. Mol Cell Endocrinol 2008; 280: 39-46.

24 Haluzik M, Dietz KR, Kim JK et al: Adrenalectomy improves diabetes in A-ZIP/F-1 lipoatrophic mice by increasing both liver and muscle insulin sensitivity. Diabetes 2002; 51: 2113-2118.

25 Hsu HT, Chang YC, Chiu YN, Liu CL, Chang KJ, Guo IC: Leptin interferes with adrenocorticotropin $/ 3^{\prime}, 5^{\prime}$-cyclic adenosine monophosphate (cAMP) signaling, possibly through a Janus kinase 2-phosphatidylinositol 3-kinase/Akt-phosphodiesterase 3-cAMP pathway, to down-regulate cholesterol side-chain cleavage cytochrome P450 enzyme in human adrenocortical NCI-H295 cell line. J Clin Endocrinol Metab 2006; 91: 2761-2769.

Supplementary Information accompanies the paper on European Journal of Human Genetics website (http://www.nature.com/ejhg) 